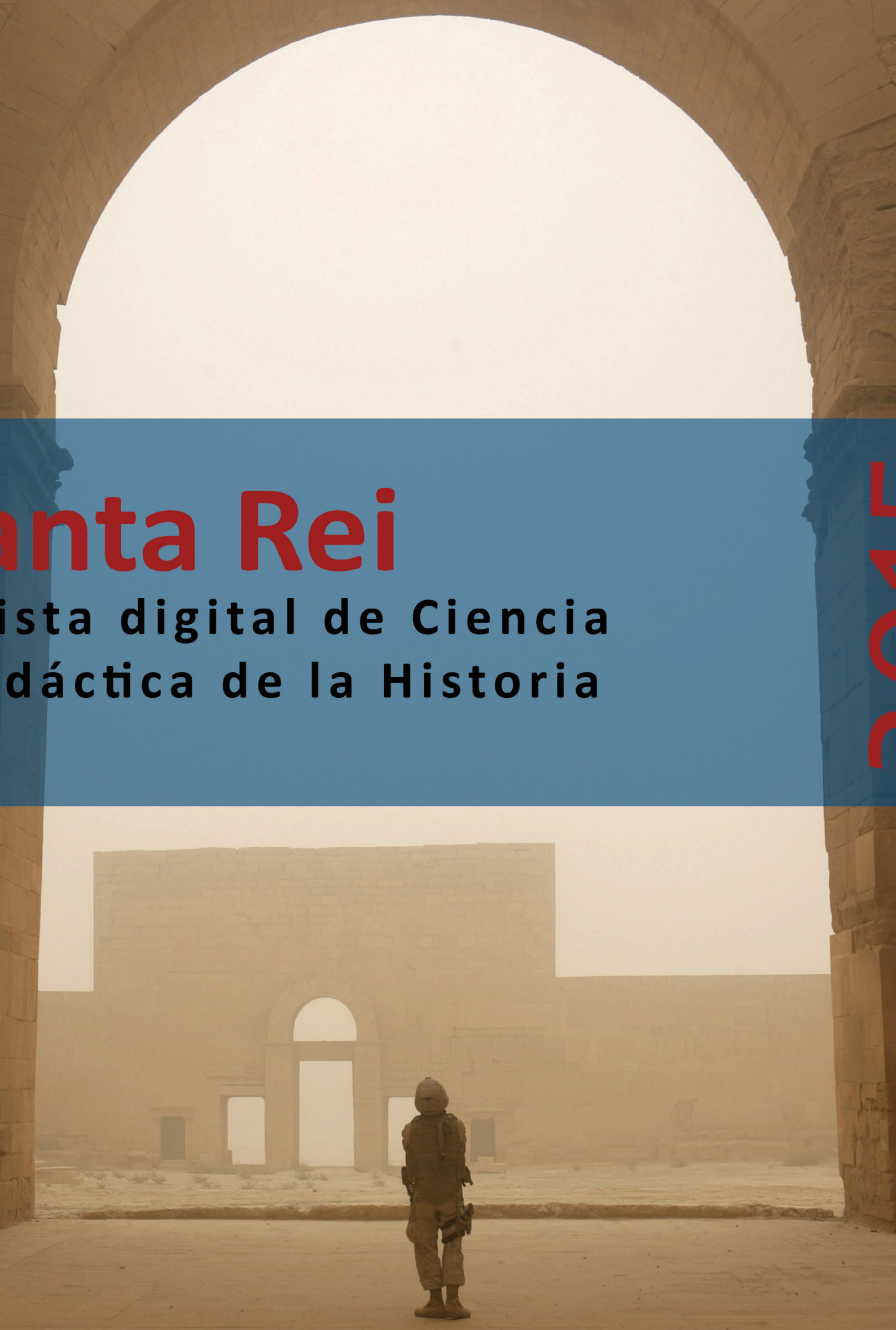

Revista digital de Ciencia y Didáctica de la Historia 


\section{Panta Rei \\ Revista Digital de Ciencia \\ y Didáctica de la Historia}

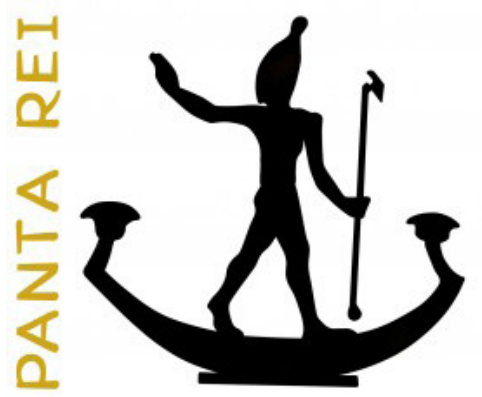

\section{5}

Revista anual

Fecha de inicio: 1995

Revista Panta Rei. pantarei@um.es

Edita:

Centro de Estudios del Próximo Oriente y la Antigüedad Tardía - CEPOAT

Edificio Universitario Saavedra Fajardo.

Universidad de Murcia

C/ Actor Isidoro Máiquez, 9

30007 - MURCIA - ESPAÑA

Teléfono: (+34) 868883890

cepoat@um.es

Web: www.um.es/cepoat/pantarei

Edición 2015

ISSNe: 2386-8864

ISSN: 1136-2464

Depósito legal: MU-966-1995
cepoAt

UNIVERSIDAD DE MURCIA centro de estudios del próximo oriente y la antigüedad tardía

En Portada: Ruinas de Hatra (Irak) (Fotografía de: Lance Cpl. Albert F. Hunt, U. S. Marine Corps).

Responsables de los textos: Sus autores.

Responsable de la presente edición: Consejo Editorial Panta Rei. 


\section{CONSEJO DE REDACCIÓN}

\section{Coordinador editorial}

Egea Vivancos, Alejandro

[Didáctica de las Ciencias Sociales, UMU]

\section{Editores}

Botí Hernández, Juan Jesús

[CEPOAT, UMU]

Meseguer Gil, Antonio José

[CEPOAT, UMU]

Sáez Giménez, David Omar

[CEPOAT, UMU]

Sánchez Mondéjar, Celso Miguel

[CEPOAT, UMU]

\section{Secretaria}

Arias Ferrer, Laura

[Didáctica de las Ciencias Sociales, UMU]

\section{Responsable informático}

Martínez García, José Javier

[CEPOAT, UMU]

\section{Traducción y corrección lingüística}

Martínez Martínez, Cristina

[Sociedad Española de Lenguas Modernas]

\section{CONSEJO ASESOR}

Albero Muñoz, M. ${ }^{a}$ del Mar [H. ${ }^{a}$ del Arte, UMU]

Chapman, Arthur

[History Education, UCL, Reino Unido]

Cobacho López, Ángel

[Derecho, UMU]

Egea Bruno, Pedro M. ${ }^{\text {a }}$

[Historia Contemporánea, UMU]

García Atienzar, Gabriel

[Prehistoria, UA]

González Monfort, Neus

[Didáctica de las Ciencias Sociales, UAB]

Haber Uriarte, María

[Prehistoria, UMU]

Irigoyen López, Antonio

[Historia Moderna, UMU]

Mahony, Simon

[Digital Humanities, UCL, Reino Unido]

Marsilla de Pascual, Francisco Reyes

[Técnicas historiográficas, UMU]

Miralles Maldonado, José Carlos

[Filología Clásica, UMU]

Molina Gómez, José Antonio

[Historia Antigua, UMU]

Noguera Celdrán, José Miguel

[Arqueología, UMU]

Pérez Molina, Miguel Emilio

[Filología Clásica, UMU]

Prados Martínez, Fernando

[Arqueología, UA]

Sánchez Ibáñez, Raquel

[Didáctica de las Ciencias Sociales, UMU]

Sancho Gómez, Miguel Pablo

[Educación, UCAM]

Vilar García, María José

[Historia Contemporánea, UMU] 

Artículos

El patrimonio arqueológico iraquí y su destrucción a lo largo del tiempo.

Fernando Espejel Arroyo.

Tacita Muta y el silencio femenino como arma del patriarcado romano.

Sara Casamayor Mancisidor.

Espartaco en la arqueología.

Juan Luis Posadas.

La batalla de Andagoste.

Luis Amela Valverde.

Las trabas para una formación arqueológica inclusiva.

Ana Samaniego Espinosa.

Repensando las narrativas nacionales: Un análisis del origen, transmisión e influencia en el aprendizaje histórico.

César López Rodríguez

El profesor como predicador, provocador y ermitaño. Un pre-texto para pensar las tareas de la didáctica de la historia.

Enrique Ibáñez Rojo

Entrevista

Evaluando el desarrollo de la competencia histórica en la enseñanza de la Historia en España. Entrevista a Jesús Domínguez Castillo.

María José Morales Rodríguez.

Reseñas

II Congreso de Jóvenes Investigadores del Mundo Antiguo de la Universidad de Murcia.

Pedro David Conesa Navarro y Juana M. ${ }^{a}$ Martínez Molina.

II Jornadas Internacionales de Didáctica de la Historia.

Eusebio Manuel Vidal Abellán.

Dölger, F. J. (2015). La luz de Cristo. Madrid: Ediciones Encuentro. 98 págs.

Alejandro Ruiz Sánchez.

Cooper, H. (ed.) (2013). Teaching History Creatively. Londres: Routledge. 185 págs.

Marta Gil de Sola Bellas.

El arte paleolítico en El Carnicero de Chabrol.

Alberto Lombo Montañés.

Normas de publicación/Publishing rules 



\title{
Repensando las narrativas nacionales: Un análisis del origen, transmisión e influencia en el aprendizaje histórico
}

\author{
Re-thinking national narratives: An analysis of their origins, \\ transmission and influence on historical learning
}

\author{
César López Rodríguez ${ }^{1}$ \\ Universidad Europea de Madrid
}

Recibido: 09/03/15

Aceptado: $17 / 03 / 15$

Para citar este artículo: López Rodríguez, C. (2015). Repensando las narrativas nacionales: Un análisis del origen, transmisión e influencia en el aprendizaje histórico. Panta Rei. Revista Digital de Ciencia y Didáctica de la Historia, 77-92.

ISSNe: 2386-8864

DOI: http://dx.doi.org/10.6018/pantarei/2015/6

\section{Resumen}

El formato narrativo ocupa un papel central en el ámbito de la historia, tanto en la generación del conocimiento histórico como en su transmisión y aprendizaje. Sin duda, una de las narrativas más frecuentes y dominantes en el campo son las denominadas narrativas nacionales. Estas narrativas, frecuentemente basadas en mitos y leyendas, buscan principalmente celebrar el pasado nacional, dificultando en gran medida una comprensión crítica del mismo. El presente artículo pretende contribuir a comprender el papel que juegan estas narrativas nacionales en la producción y el consumo del conocimiento histórico a través de un análisis en diferentes ámbitos: el académico, el escolar y el ámbito informal. Finalmente, se presenta una reflexión sobre algunos de los estudios empíricos más recientes que analizan la influencia de estas narrativas nacionales en el aprendizaje de los estudiantes.

\section{Palabras clave}

Narrativas Nacionales, Identidad Nacional, Aprendizaje Histórico, Nacionalismo Banal.

\begin{abstract}
The narrative format plays a central role in the field of history, both in the production of historical knowledge and its transmission and also when it comes to learning. One of the most common and dominant narratives are the so-called national narratives. These narratives, frequently based on myths and legends, aim to celebrate the nation and hinder a critical understanding of the past. This article seeks a better understanding of the role that these national narratives play in the production and consumption of historical knowledge through an analysis in different contexts: academic, school and at an informal ambit. Finally, some of the most recent empirical studies on the influence of these
\end{abstract}

1 Para contactar con el autor: César López Rodríguez. Universidad Europea de Madrid. Departamento de Psicología. C/ Tajo s/n, Villaviciosa de Odón, 28670 Madrid. cesar.lopez2@uem.es. 
national narratives on students learning are discussed.

\author{
Keywords \\ National Narratives, National Identity, History Learning, Banal Nationalism
}

\title{
1. Introducción ${ }^{2}$
}

La narración, más allá del campo de la historia, constituye no solamente un tipo discursivo, una configuración textual determinada, sino también un modo específicamente humano de organizar el pensamiento. Los seres humanos interpretamos narrativamente tanto nuestras acciones y comportamientos como las de los demás, existiendo por tanto, una predisposición a organizar la experiencia de forma narrativa, mediante estructuras de tramas que dotan de significado a dicha experiencia (Bruner, 1990). Autores como Egan (1997), sostienen que somos animales narradores, es decir, solemos dar sentido a las cosas en forma narrativa. Para Barton y Levstik (2004) las narrativas son una poderosa herramienta cultural para la comprensión de la historia. Este formato narrativo está presente en muchas de las maneras a través de las que nos acercamos al pasado: cuentos, mitos, novelas, museos, diarios o películas son algunas de ellas. Los libros de texto utilizados en muchos colegios sin duda también. Como señala Von Borries (2009), la estructura narrativa es una condición inevitable de la historia.

Desde una edad muy temprana estamos rodeados por fuentes que poseen una estructura narrativa, aprendemos a usarlas desde pequeños e incluso sabemos qué podemos esperar de ellas. Un buen número de estudios reflejan que las historias se recuerdan mejor cuando poseen una estructura típicamente narrativa (Mandler, 1984). Incluso, podemos considerar que las narrativas constituyen una buena herramienta para que nuestros alumnos manejen conceptos tan centrales en el aprendizaje de la historia como son las relaciones causales y la temporalidad. Por todo esto, no es de extrañar que algunos educadores equiparen la enseñanza de la historia con contar una buena narrativa (Barton y Levstik, 2004; VanSledright, 2008). Sin embargo, pese a la existencia de las mencionadas ventajas del pensamiento narrativo en el ámbito de la historia, conviene tener en cuenta también determinadas características del mismo que en muchas ocasiones dificultan el desarrollo de un pensamiento histórico. Es importante recordar que, pese a que el conocimiento histórico toma en muchas ocasiones un formato narrativo, no podemos identificar historia y narrativa como una misma cosa. Las narrativas constituyen una herramienta más para acercarnos al conocimiento del pasado, pero no son el pasado en sí (Carretero y López, 2010). Ahora bien, ¿qué características tiene esta herramienta y cómo influye en la comprensión del pasado?

Algunos trabajos clásicos realizados desde la psicología social presentan aportaciones muy relevantes que pueden ayudarnos a responder esta pregunta. Especialmente interesantes son los trabajos de Bartlett (1932) sobre la influencia de las narrativas en el recuerdo. Bartlett puso de relieve cómo las personas no recordamos elementos del pasado de manera discreta o aislada, sino que aplicamos "esquemas" aprendidos en nuestra experiencia sobre esos elementos para integrarlos y recordarlos. En su trabajo más conocido Bartlett (1932) analizó el modo en el que estudiantes de Cambridge recordaban una historia tradicional de los nativos americanos denominada la Guerra de los Fantasmas (The War of the Ghosts). El estudio mostró cómo los estudiantes, en sucesivos ensayos de recuerdo, fueron transformando, y en cierta manera re-inventando, la historia original aplicando elementos de su contexto cultural inglés. Así, las canoas indias pasaron a ser barcos, los nombres indios desaparecían y los elementos sobrenaturales a los que hacía referencia la historia

2 Este artículo ha sido elaborado con la ayuda del Proyecto "Representación de narrativas históricas en distintos entornos culturales" (EDU2013-42531-P) dirigido por Mario Carretero y financiado por el Ministerio de Economía y Competitividad. 
fueron racionalizados. Como comenta Bartlett, la historia folclórica india se "convencionalizó". Es decir, los participantes del estudio, a medida que pasaba el tiempo y se les pedía que volvieran a recordar la historia, iban aplicando "esquemas" de su propio contexto y experiencia para generar una narrativa. Los elementos que no encajaban con ese esquema narrativo -como podían ser las canoas o los nombres indios- iban desapareciendo, dando lugar a una narrativa muy diferente a la original. En conclusión, cuando recordamos el pasado, ponemos en marcha una serie de esquemas narrativos generados en el contexto social y cultural presente que influyen enormemente sobre nuestro recuerdo.

Esta idea es retomada por Wertsch (2004) en su distinción entre esquemas narrativos maestros y narrativas específicas. Los esquemas narrativos maestros constituirían una guía o modelo general para las narrativas específicas. Dicho de otro modo, diferentes narrativas específicas comparten determinadas características globales que encajan dentro de ese esquema narrativo maestro. Es importante destacar que estos esquemas narrativos maestros son construidos en un contexto social y compartidos por los miembros de un grupo, pudiendo variar entre diferentes grupos sociales. De este modo, los individuos acudirían a los esquemas narrativos maestros a la hora de generar sus propias narrativas específicas para interpretar el pasado, como ocurría con los estudiantes de Cambridge. Este proceso se produce en numerosas ocasiones de manera implícita y automática. Así, los esquemas narrativos maestros funcionarían de modo similar a teorías implícitas, en el sentido de que rara vez son cuestionados e incorporan creencias muy arraigadas (Ross, 1989). El propio Wertsch (2004) ejemplifica esta influencia de las narrativas maestras para la construcción de narrativas específicas aplicado al caso de la historia rusa. Wertsch identifica la narrativa maestra que denomina "triunfo sobre las fuerzas extranjeras". Esta narrativa maestra serviría para generar narrativas específicas sobre diferentes eventos de la historia rusa, como por ejemplo: la invasión de los mongoles, las invasiones napoleónicas o la invasión alemana durante la Segunda Guerra Mundial. Barton y Levstik (2004) señalan, por su parte, la existencia de una narrativa maestra para el caso estadounidense que denominan "búsqueda de libertad y progreso" y que se encuentra presente en muchas de las narrativas específicas de la historia de Estados Unidos. Así, por ejemplo, narrativas históricas específicas como la conquista del Oeste, la intervención del país en la Primera y Segunda Guerra Mundial o la Guerra de Vietnam son interpretadas y generadas atendiendo a este esquema general maestro de "búsqueda de libertad y progreso". Pero más allá de estos ejemplos concretos de narrativas maestras, el esquema narrativo maestro por excelencia y uno de los más presentes, repetidos e influyentes en el ámbito de la historia es el que constituyen las denominadas narrativas nacionales.

\section{Narrativas nacionales como narrativas maestras en Historia}

Sin duda, las narrativas más frecuentes en el ámbito de la historia son las narrativas nacionales (Barton y Levstik, 2004; Barton y McCully, 2005; Carretero, 2011; Carretero y López, 2010). En cualquier biblioteca o librería que contenga obras relativas al estudio de la historia podemos encontrar numerosos estantes repletos de títulos como: Historia de España, Historia de Inglaterra, Historia de Estados Unidos y de casi cualquier nación que podamos imaginar. Estos libros normalmente reflejan la Historia oficial de cada nación en un formato narrativo que abarca desde sus orígenes -normalmente situado en tiempos inmemoriales- hasta la actualidad. En general, las narrativas nacionales poseen fundamentalmente unas fuertes características de tipo romántico. Se trata de narraciones cerradas que tratan de dibujar una clara línea entre el pasado, el presente y el futuro, haciendo a la nación una perenne protagonista. Como señala Balibar (1991, p. 135):

La historia de las naciones, empezando por la nuestra, se nos ha presentado siempre con las características de un relato que les atribuye la continuidad de un sujeto. De este modo, la formación de la nación aparece como la culminación de un "proyecto" secular, jalonado de etapas y de tomas de conciencia que los perjuicios de 
los historiadores presentarán como más o menos decisivas, pero que, de todas formas, se inscriben en un esquema idéntico: el de la manifestación de la personalidad nacional.

Estas narrativas nacionales, entendidas como una narrativa maestra, poseen determinadas características generales que podemos encontrar en muchas de las narrativas específicas nacionales de muy diversos países (Carretero y Bermúdez, 2012):

- La nación y sus habitantes son los principales protagonistas de la narrativa. Así, en numerosas ocasiones, surgen narrativas de conflicto entre el "nosotros" nacional y el "ellos" extranjero.

- Los conceptos de "nación" y de "identidad nacional" son reflejados como entidades atemporales y esencialistas que pueden ser encontrados en cada periodo de la historia.

- Las acciones del grupo nacional son siempre juzgadas moralmente de un modo positivo en contraposición a las llevadas a cabo por "los otros" extranjeros.

- En numerosas ocasiones, los conflictos sobre el territorio nacional constituyen uno de los temas principales de la narrativa. En estos conflictos, se suele legitimar la posesión atemporal del territorio por parte del grupo nacional.

Estas cuatro características giran en torno a dos aspectos centrales. Por un lado, el modo en el que se representan los conceptos de nación e identidad nacional y, por otro, los elementos emocionales y los juicios morales contenidos en la narrativa. En cuanto a los conceptos de nación e identidad nacional, es importante recalcar su papel en estas narrativas de protagonistas indiscutibles. La característica quizás más influyente de estos conceptos es su supuesto carácter atemporal y esencial. Es decir, la gran mayoría de narrativas nacionales cuentan una serie de eventos sucedidos a lo largo del tiempo que tienen como protagonista de fondo a la nación. Una nación que permanece como entidad atemporal y cuyos habitantes son portadores de una esencia identitaria, la identidad nacional. Así, la nación y la identidad nacional se presentan como elementos naturales del ser humano. El objetivo final es establecer un vínculo entre el pasado, el presente y el futuro que gira alrededor de estos dos conceptos, la nación y la identidad nacional (Barton, 2009). Con ello se procura que el lector en el presente se vincule e identifique con la historia de su nación y de aquellos que supuestamente siempre han compartido su misma identidad nacional.

Asimismo, merece la pena señalar que estas narrativas, como herramienta organizadora del conocimiento, están cargadas de elementos emocionales e identitarios (Barton y Levstik, 2004; Rüsen, 2004). Solemos atribuir intenciones, juicios morales y de valor a los protagonistas de las narrativas y también solemos identificarnos con ciertos protagonistas de las mismas y no con otros. Las narrativas y los contenidos históricos específicamente generan muy a menudo respuestas y juicios morales. Solemos enjuiciar las acciones del pasado como "justas" o "injustas", como "admirables" o "reprobables", y calificar a los protagonistas de estas acciones como "héroes" o "villanos". Como comentan Barton y Levstik (2004), posicionarnos moralmente es algo ineludible cuando nos encontramos con el pasado. Las narrativas nacionales suelen valorar en términos positivos las acciones del propio grupo nacional, fundamentando sus argumentos en términos esencialistas y no históricos. En ellas, se recuperan de manera acrítica eventos históricos y sus personajes, que en muchas ocasiones son transformados en héroes nacionales. Las fuentes que ponen en conflicto una visión complaciente de la propia nación suelen ser rechazadas u olvidadas (Carretero y Montanero, 2008). Como ya señalaba Renan (1882), el olvido, e incluso el error histórico, son un factor esencial en la creación de una nación. No es de extrañar, por tanto, que el progreso de los estudios históricos que revisan críticamente estas narrativas nacionales románticas sea visto como un peligro para las mismas (Foster, 2012).

Las narrativas nacionales, por lo tanto, poseen unas características que parecen favorecer más la creación de una identidad nacional en el lector que la adquisición de un pensamiento histórico que permita acercarnos a la comprensión del pasado. La dificultad de desarrollar un pensamiento histórico crítico basado en las narrativas nacionales resulta más evidente si tenemos en cuenta que las pretensiones de atemporalidad, permanencia y naturalidad de la nación y la identidad nacional son, desde el punto de vista historiográfico actual, rotundamente falsas (Hobsbawm, 1997; Smith, 
1991).

Si queremos comprender la influencia que tienen estas narrativas nacionales en la generación del conocimiento histórico y en el aprendizaje de la historia, conviene hacer algunas reflexiones. ¿De dónde surgen estas narrativas nacionales? ¿Qué objetivos tienen? ¿Por qué se perpetúan? ¿Qué presencia tienen en los distintos ámbitos en los que se transmite la historia? ¿Cómo influyen en el aprendizaje de los propios estudiantes? Para tratar de responder a estas preguntas, en el presente artículo se presenta un análisis de las narrativas nacionales en tres planos especialmente relevantes: la propia historiografía académica, el ámbito escolar, y el contexto informal. Por último, se hará referencia a algunos de los recientes estudios que, de manera empírica, se han desarrollado para entender mejor la influencia de las narrativas nacionales en el aprendizaje de los estudiantes.

\subsection{Las Narrativas Nacionales en la Historiografía: El Ámbito Académico}

Lorenz (2011) señala la existencia de una fuerte tensión en la disciplina desde sus inicios. Esta tensión se produce entre dos pretensiones fundamentales de la historia académica: La pretensión de ser científica y la pretensión de ser práctica. La pretensión científica busca desterrar los mitos sobre el pasado y reemplazarlos con "La Verdad". La pretensión práctica, por su parte, busca otorgar un sentido aplicado a la vida diaria, es decir, una especie de guía práctica. Como señalan diversos autores, para muchos historiadores de los siglos XIX y XX, esta vertiente práctica ha consistido fundamentalmente en crear un sentimiento de identidad entre el ciudadano y la nación (Anderson, 1983; Hobsbawm, 1997; Renan, 1882). No es de extrañar que los historiadores hayan sido considerados muchas veces como los "sacerdotes del estado" (Lorenz, 2011).

Para analizar el origen de las narrativas nacionales es importante retroceder a los inicios de la propia historiografía. La historia que surge como disciplina moderna a finales del siglo XVIII y principios del XIX estuvo marcada fuertemente por un contexto abiertamente nacionalista. Sus principales propósitos giraban en torno a la legitimación de las naciones que emergían en ese momento y a la construcción de una identidad nacional en los nuevos ciudadanos (Anderson, 1983; Cruz, 2005; Wallerstein, 1991). Para ello, la historiografía del siglo XIX nacionalizó la historia de cada una de las naciones estableciendo un vínculo desde el pasado más remoto hasta el presente nacional (Balibar, 1991). Se pretendía dotar a la nación y a sus miembros de una antigüedad inmemorial y natural que legitimase el nuevo orden político. Con este fin, la propia historiografía de cada nación produjo una narrativa que recogía el devenir de la nación desde la antigüedad hasta su apogeo (Symcox y Wilschut, 2009). Estas narrativas nacionales, más fundamentadas en mitos y leyendas que en hechos históricos, se perpetuaron como verdades incuestionables del pasado de cada nación (Ballantyne, 2005). Desde este enfoque tradicional, catalogado como primordialista (Ichijo y Uzelac, 2005), la historia se construye hacia atrás, con una clara intención de nacionalizar el pasado. La gran mayoría de eventos son nacionalizados. A los antiguos pueblos, personajes y regiones del pasado se les atribuye la característica de nacional. Los grandes héroes del pasado dejan de pertenecer a otros pueblos o sociedades para convertirse en héroes nacionales (Pérez, 2001). Lo mismo ocurre con el territorio, ya no es cualquier territorio el que está en juego, sino que es el territorio nacional -casualmente coincidente con el actual. De este modo la historiografía tradicional legitimaba claramente a las naciones que surgían en ese momento como algo natural, otorgándolas un carácter atemporal y eterno (Gellner, 1983).

Sin embargo, desde finales del siglo XX aparece dentro de las disciplinas de la historia, la sociología y las ciencias políticas, un nuevo enfoque que pone de manifiesto el carácter construido y moderno del fenómeno nacional (Álvarez, 2001). Este enfoque, denominado enfoque modernista (Ichijo y Uzelac, 2005), sitúa el surgimiento de las naciones en un momento determinado de la historia, precisamente en la era de los nacionalismos, entre finales del siglo XVIII y el siglo XIX (Connor, 2004; Hobsbawm, 1997). Desde este punto de vista, las naciones no son una entidad natural, sino una construcción social de época reciente. Como recoge la célebre frase de Gellner, "es el nacionalismo el que crea las naciones, y no al revés" (1983, p. 55). En la misma línea apuntan autores clásicos 
como Hobsbawm o Anderson, cuando hablan del carácter "inventado" o "imaginado" de la nación, desmitificando radicalmente el carácter "natural" (Anderson, 1983; Hobsbawm y Ranger, 1983).

Pese a que en la actualidad aún podemos encontrar ambos enfoques, podemos considerar que la aproximación primordialista es característica de la historiografía romántica de los siglos XVIII, XIX y gran parte del $X X$, mientras que el enfoque modernista se ha impuesto como enfoque disciplinar desde finales del siglo XX. Álvarez recoge este giro dentro de la propia historiografía (2001, p. 15):

En vez de aceptar las identidades nacionales como realidades naturales, comenzaron a verse como creaciones artificiales, movidas por intereses políticos. El sentimiento nacional, en lugar de creerse espontáneo o innato, pasó a considerarse adquirido o inculcado, fundamentalmente a través del proceso educativo, pero también por medio de ceremonias, monumentos o fiestas cívicas. Se cayó en la cuenta de que los estados, tenidos hasta entonces por invenciones humanas que se apoyaban en fenómenos sociales y culturales previos, eran los promotores del proceso; lo político precedía a lo étnico, y no al contrario. Se comprendió también que las identidades nacionales eran cambiantes, en lugar de permanentes.

Este cambio en la manera de entender estos conceptos supone una revolución en el modo de entender la propia historia y, por supuesto, las narrativas nacionales. El conflicto entre enfoques es evidente. Para el enfoque primordialista toda la historia, desde el más remoto pasado, está estructurada en torno a la nación. Desde el enfoque modernista actual la mayoría de historiadores defiende que no se puede hablar de naciones en épocas anteriores a la era de los nacionalismos (Cruz, 2005). Como hemos visto, la historiografía romántica nacionalizó el pasado, reinterpretándolo desde el punto de vista de cada una de las naciones y plasmándolo en narrativas nacionales excluyentes que se perpetuaron como versiones únicas de la historia. Para el enfoque disciplinar actual, dichas narrativas nacionales constituyen más una visión mítica que una interpretación historiográfica del pasado. Por lo tanto, desnacionalizar los análisis históricos de épocas anteriores al surgimiento de las naciones constituye uno de los objetivos de este nuevo enfoque (Foster, 2012).

Es importante destacar que la predominancia actual del enfoque modernista no implica la desaparición, ni mucho menos, del enfoque primordialista en el ámbito académico. Es frecuente encontrar historiadores defendiendo argumentos o posiciones cercanas al punto de vista primordial en encendidos debates políticos sobre los usos públicos de la historia (Carretero, 2011; Nakou y Barca, 2010). Este fenómeno ocurre con más frecuencia cuando estos debates tienen que ver con el ámbito educativo y con el problema de qué historia se debe enseñar en las escuelas y con qué fines.

\subsection{Narrativas Nacionales, el Currículo y los Libros de Texto: El Ámbito Escolar}

La aparente superación de enfoques identitarios y primordialistas sobre el fenómeno nacional dentro del ámbito académico contrasta fuertemente con la omnipresencia de las narrativas nacionales en las escuelas de la gran mayoría de países (Foster, 2012; Grever y Stuurman, 2007; Symcox y Wilschut, 2009). Pese a su carácter ahistórico y muchas veces mítico, estas narrativas nacionales toman un papel central en el currículo. Así, Paxton (1999) y Alridge (2006), a partir de un exhaustivo análisis de los libros de texto estadounidenses, ponen de manifiesto que las narrativas sobre los «grandes» hombres y los acontecimientos que guiaron a Estados Unidos hacia un ideal de progreso y civilización continúa siendo la manera prototípica mediante la cual muchos historiadores y libros de texto difunden el conocimiento histórico. Como señala Ballantyne (2005), estas narrativas, ya a principios del siglo XX, se constituyeron en tradiciones de la historia nacional que -como indica en lo que parece una acertada metáfora- calcificaron en muchos países de Europa, así como en las colonias europeas y gran parte de Asia (Duara, 1995). Estas narrativas nacionales reflejan el concepto anteriormente comentado de esquema narrativo maestro (Werstch, 2004). Son 
narrativas construidas y compartidas socialmente, aceptadas por la gran mayoría de la sociedad como verdades naturales incuestionables.

En el campo de la enseñanza de la historia, un elemento clave a la hora de recoger y transmitir estas narrativas históricas es el libro de texto. De acuerdo con Foster (2012, p. 49), "Ios libros de texto de historia en muchas naciones a lo largo del mundo están marcados por dos características. La primera es que son abiertamente nacionalistas. La segunda es que normalmente reflejan una única historia oficial de estilo narrativo." ${ }^{3}$. Foster (2006) señala que en las últimas cuatro décadas los libros de texto estadounidenses se han caracterizado por un énfasis en el estado-nación, en el que el objetivo no era examinar o reexaminar el pasado, sino celebrar los logros de la nación. Para ello frecuentemente se minimiza la larga historia de conflictos intergrupales y étnicos de los Estados Unidos, con la finalidad de fomentar una unión nacional.

En esta misma línea Finn (2007), hablando del caso ruso en un artículo del Washington Post, comentaba: "con dos nuevos manuales de historia y ciencias sociales para la enseñanza superior en la escuela, escritos por consultores políticos del Kremlin, las autoridades rusas tratan de imbuir el debate en la clase con un punto de vista nacionalista"2 (para. 1). Janmaat (2005) señala cómo en países de la antigua Unión Soviética como Ucrania, los libros de texto proveen a los constructores del sentimiento nacional con una infinita fuente de materiales basados en mitos y narrativas históricas para fundamentar un discurso nacionalista. En Taiwán nos encontramos con un caso similar. Hasta el año 2002, se utilizaba un único libro de texto estándar para la enseñanza de la historia. Desde entonces, las escuelas pueden elegir entre cuatro o cinco libros de texto distintos escritos de acuerdo a una guía nacional. Pese a que los profesores pueden utilizar otros materiales en las clases, el gobierno regula la publicación de los libros de texto y los contenidos que tienen que ser memorizados por los alumnos (Hsiao, 2005).

La presencia de la nación y de contenidos relacionados con ésta en el currículo de historia en la gran mayoría de los países europeos sigue muy presente en la actualidad. La asociación EUROCLIO, interesada en fomentar el desarrollo de un enfoque innovador e inclusivo de la historia y la educación de la ciudadanía en Europa (pero también en crear una identidad de carácter europeo), pone de manifiesto la importancia de las naciones en la enseñanza de la historia. De este modo, cuando en 2003 se llevó a cabo un análisis sobre qué dimensión había aumentado más su presencia en el currículo desde 1980 (nacional, regional, europea o mundial), la historia nacional resultó ser la que más había aumentado (Van der Leeuw-Roord, 2004, 2007). La idea que se desprende es que la fundamentación nacional de los currículos y libros de texto en Europa no ha cambiado demasiado desde 1989. Incluso se puede apreciar un aumento de los enfoques fundamentados en la nación, recurriendo a una metodología tradicional de la enseñanza de la historia (Mak, 2005). Como señaló Van der Leeuw-Roord (2007), en 2008 la enseñanza de la historia nacional aparecía como un contenido central en la agenda de muchos países europeos. Desde diversos ámbitos -el político, los medios de comunicación e incluso los propios historiadores- se hace hincapié en que el público general tiene una falta de conocimiento histórico nacional. Para solventar esta carencia, en un grupo creciente de países, los políticos abogan por un incremento de la historia nacional en el currículo.

En países como Holanda, los debates sobre el currículo de historia han llevado a introducir el término canon para describir el conocimiento obligatorio que los estudiantes deben aprender sobre la historia y la cultura de Holanda (Grever, 2006). El canon aplicado a la historia defiende la idea de una gran narrativa histórica, donde se seleccionan determinadas figuras, eventos, ideas y valores, en defensa de unas determinadas perspectivas y explicaciones (Grever y Stuurman, 2007). Como señala Van der Leeuw-Roord (2004), el objetivo explícito de la introducción de un canon nacional sobre el conocimiento histórico no es otro que fortalecer la memoria colectiva de Holanda. Incluso en Gran Bretaña, uno de los principales focos del surgimiento del enfoque modernista de la enseñanza de la historia, el secretario de educación en 2005, Tim Collins, defendía la idea de crear un nuevo currículo de historia basado en la herencia común, que hiciera posible la supervivencia

3 En inglés en el original. Traducción propia.

Panta Rei (2015), 77 - 92 
de la nación británica (Grever y Stuurman, 2007). En la misma línea, en 2012, el Secretario de Educación, Michael Gove, abogaba por que los estudiantes adquirieran una "narrativa lineal de la historia británica" (Vasagar, 2012, par. 8).

En el ámbito español, cabe recordar la célebre y polémica frase del ministro de educación, José Ignacio Wert, en el Congreso de los Diputados en 2012:

Nuestro interés es españolizar a los alumnos catalanes y que se sientan tan orgullosos de ser españoles como de ser catalanes.

(Aunión, 2012, par. 1)

Más allá de las interpretaciones realizadas desde sectores españolistas y catalanistas, la intervención refleja un claro vínculo entre la educación y la formación de identidades nacionales.

Sin embargo, no en todos los países el currículo y los libros de texto presentan una narrativa cerrada con objetivos fundamentalmente identitarios. En Inglaterra, por ejemplo, pese al énfasis que desde el gobierno se pretende hacer en una narrativa oficial, existen esfuerzos por entender el libro de texto como una fuente más a la hora de enseñar la asignatura en las escuelas. Se trata de ofrecer una herramienta que favorezca la comprensión del carácter interpretativo y en muchas ocasiones conflictivo de la historia (Foster, 2012). No obstante, este enfoque disciplinar en Inglaterra parece ser más la excepción que la norma entre las naciones-estado (VanSledright, 2008; Van Der Leeuw-Roord, 2009).

La fuerte presencia de contenidos nacionales e identitarios en las narrativas nacionales de numerosos países debe hacernos reflexionar sobre el impacto que pueden tener en el aprendizaje. La historia formal, tanto el ámbito académico como el ámbito escolar, juega sin lugar a dudas un importante papel en la transmisión de la historia nacional. Sin embargo, no debemos olvidar cómo, cada día más, otros medios y géneros que encontramos en el ámbito informal tienen una mayor influencia en cómo se producen y se consumen las narrativas nacionales.

\subsection{Narrativas Nacionales y los Usos Públicos de la Historia: El Ámbito Informal}

Los textos escolares no son el único medio a través del cual se da forma a la identidad nacional y se transmiten narrativas nacionales (Berger, Eriksonas y Mycock, 2011). En muchas ocasiones estas narrativas se ven reforzadas a través de novelas de divulgación histórica, museos (Asensio y Pol, 2012; González de Oleaga, 2012), películas (Wineburg, Mosborg y Porat, 2001), memoriales públicos (Wineburg, Mosborg, Porat y Duncan, 2007) o en celebraciones y efemérides patrias ${ }^{4}$.

Centrémonos, por ejemplo, en las celebraciones y efemérides nacionales. No nos será difícil encontrar ejemplos de estas festividades en muy diversos países. Muchas de ellas tienen que ver con señalados eventos del glorioso pasado nacional, como los que hacen referencia a revoluciones, grandes batallas o procesos de independencia nacional. El famoso 4 de julio estadounidense, el 14 de julio francés o el 12 de octubre en España serían ejemplos de estas efemérides. Año tras año se recuerda este vínculo entre el presente nacional y su glorioso pasado. Nuevamente, el objetivo es reforzar el vínculo atemporal entre los ciudadanos del presente y un pasado remoto recurriendo a la supuesta identidad nacional común (Carretero, 2011). En muchas ocasiones aparece una narrativa asociada a estas festividades que en gran medida recupera esa narrativa nacional. Así, por ejemplo, el día 12 de octubre en España conmemora la narrativa del llamado "Descubrimiento" de América como uno de los hitos del pasado nacional. La festividad estadounidense del 4 de julio, por su parte, rememora el inicio de la Guerra de la Independencia Americana. Estas festividades son un modo de revivir la historia y volver a contar determinadas narrativas que se perpetúan año tras año. Sin embargo, el objetivo de estas fiestas parece ser más el de celebrar la nación y su pasado que el de realizar una mirada crítica para entender el mismo. Son celebraciones que

4 Para un exhaustivo análisis de estas prácticas en el caso de Argentina véase Carretero y Kriger (2006). 
mayormente buscan una exaltación del sentimiento nacional y que normalmente poco o nada tienen que ver con comprender lo que sucedió en ese acontecimiento histórico concreto. Al contrario, en estas celebraciones muchas veces se recrean y refuerzan elementos míticos -incluso totalmente inventados- del pasado nacional que conforman elementos clave de la narrativa nacional oficial.

Algo similar ocurre con muchos de los memoriales con que nos encontramos si paseamos por nuestras ciudades (Valsiner, 2012; Wineburg, Mosborg, Porat y Duncan, 2007). Estos monumentos con los que convivimos día a día y ante los que pasamos en muchas ocasiones casi sin prestar atención también están contando una historia. Quizás seamos más conscientes de este fenómeno cuando visitamos alguna ciudad como turistas y vamos reparando de manera explícita en los monumentos y memoriales. Trafalgar Square en Londres, el Arco del Triunfo de París o The National Mall en Washington D.C., donde podemos encontrar los memoriales de Lincoln, el propio Washington o el de las guerras de Vietnam o Corea, serían algunos de los ejemplos más famosos. La gran mayoría de estos monumentos buscan reflejar y glorificar esa historia nacional y, por tanto, refuerzan esa narrativa nacional oficial en lugar de suponer una mirada crítica al pasado. Si pensamos en nuestra propia ciudad es posible que nos vengan a la mente monumentos que conmemoran esos hitos gloriosos del pasado nacional. Sin embargo, estos monumentos, al igual que ocurre muchas veces con las narrativas que encontramos en los libros de texto, destacan o conmemoran la historia nacional de una manera sesgada. Este sesgo, obviamente, suele producirse a favor del propio grupo nacional. Se deciden celebrar unos eventos pero no otros y lo mismo ocurre con los personajes históricos. Así, por ejemplo, en España encontramos numerosos monumentos que ensalzan el hito del denominado "Descubrimiento" de América en la figura de Cristóbal Colón. Por su parte, esta figura histórica, que podría ser considerada por muchos españoles como un héroe del pasado nacional, es cada vez más denostada y fuertemente criticada al otro lado del Atlántico. Muchas son las voces críticas que se levantan contra las celebraciones que, como el "Columbus Day", conmemoran la llegada de los europeos y la figura de Colón. Este fenómeno no debería extrañarnos si tenemos en cuenta que la narrativa oficial que se maneja de este acontecimiento en países como España y Méjico son muy diferentes (Carretero, Jacott y López-Manjón, 2002).

En definitiva, muchos de los monumentos, los nombres de las calles y plazas por las que paseamos e incluso muchas festividades del calendario, nos acercan hacia el pasado recordándonos que somos miembros de una nación y refuerzan en nuestra memoria esa narrativa nacional. Este fenómeno es lo que Billig (1995) ha denominado nacionalismo banal. Billig defiende la tesis de que, aunque a veces las manifestaciones explícitas del nacionalismo son vistas como un fenómeno superado y perteneciente al pasado, otras formas más implícitas con las que convivimos en el día a día, como las anteriormente mencionadas, refuerzan estas mismas ideas. Este nacionalismo banal del que habla Billig enlaza con la idea de Werstch (2004) expuesta al comienzo de este artículo de los esquemas narrativos maestros. Ambos hacen referencia a un conocimiento generado y compartido por un grupo social concreto, en este caso el grupo nacional, y que posee un carácter más bien implícito. Es decir, en muchas ocasiones no somos conscientes de estos fenómenos, que sin embargo, conforman las raíces de creencias fuertemente arraigadas que rara vez se discuten.

Por lo tanto, en muchas ocasiones, las prácticas que encontramos en el ámbito informal y que hacen referencia al pasado nacional, ya sea a través de celebraciones, novelas, series de televisión, monumentos o museos, están reforzando las tan familiares narrativas nacionales. Este respaldo a la narrativa puede producirse bien reproduciendo la propia narrativa, como puede ocurrir en una novela, en una película o en un museo, o bien reforzando algún elemento clave de la narrativa, como puede ser un personaje o un concepto central que vertebre la narrativa. Ejemplos de conceptos centrales que pueden disparar un tipo de narrativa concreto serían, por ejemplo, los conceptos de "Descubrimiento", para dar cuenta de la narrativa de la llegada de los europeos al continente americano, o el de "Reconquista", para reflejar lo ocurrido en la Península lbérica entre los siglos VIII y XV. Sin duda, el concepto central que vertebra toda narrativa nacional es el propio concepto de nación. Así, el modo en el que entendamos este concepto -bien como algo natural y permanente o más bien como un concepto reciente y socialmente construido- va a determinar en 
gran medida nuestra narrativa sobre el pasado nacional.

Ahora bien, una vez analizada la fuerte presencia que las narrativas nacionales tienen en distintos ámbitos como el académico, el escolar o el informal, cabe preguntarse: ¿De qué manera influye esto en el aprendizaje de la historia? ¿Cuál es el uso que hacen los estudiantes de estas narrativas cuando tratan de dar sentido al pasado? ¿Cómo entienden ellos conceptos centrales de estas narrativas como los de nación o identidad nacional? En el siguiente apartado trataremos de dar respuesta a estas preguntas.

\section{Aprendiendo narrativas nacionales: la visión de los estudiantes}

Pese a que son muchos los trabajos y debates que han abordado la presencia de narrativas nacionales desde un punto de vista teórico, son pocos los que lo han hecho desde el punto de vista empírico. En este apartado se recogen algunos de los trabajos más recientes que se han desarrollado con respecto al uso que hacen estudiantes de diferentes niveles educativos de las narrativas nacionales.

Uno de los aspectos que resultan más interesantes es el grado en el que los alumnos recurren a la narrativa nacional oficial para elaborar sus interpretaciones o, por el contrario, desarrollan una mirada más crítica hacia el pasado. Barton y Levstik (2004) examinaron las explicaciones causales que realizaban estudiantes estadounidenses de entre 10 y 14 años de edad a la hora de analizar la intervención de su país en la Guerra de Vietnam o las Guerras Mundiales. La idea era ver hasta qué punto los estudiantes recurrían a la narrativa nacional estadounidense de "búsqueda de libertad y progreso" tan frecuentemente encontrada en los libros de texto. Efectivamente, la gran mayoría de los estudiantes realizó interpretaciones que justificaban en todo momento la intervención de su país. Estos autores señalan que los estudiantes defendían una superioridad moral de su propia nación. Argumentos como "para ayudar a otra gente...estábamos luchando por otra gente" o "para ayudar a los vietnamitas" fueron los más comunes. Por lo tanto, las acciones de su nación quedaban legitimadas, mientras que visiones más complejas o críticas que no encajan con la narrativa oficial estadounidense prácticamente no existen. Parece que los argumentos esgrimidos por los estudiantes son congruentes con el tipo de narrativas que Alridge (2006) y Paxton (1999) encontraban en sus análisis de los libros de texto estadounidenses y que hacen referencia a la gran narrativa de "búsqueda de libertad y progreso".

En el ámbito español se han llevado a cabo estudios con estudiantes universitarios para analizar específicamente cómo éstos interpretan contenidos nacionales. En uno de ellos (López, Carretero y Rodríguez-Moneo, 2014a), el contenido analizado fue el de la denominada Reconquista. Sin duda, la Reconquista constituye una narrativa nacional que la historiografía tradicional ha considerado como fundacional de la nación Española. En ella se narra la pérdida y posterior reconquista de España en su lucha contra los musulmanes. Sin embargo, como ocurre con las grandes narrativas nacionales, diversos autores han puesto de manifiesto el carácter más mítico que historiográfico de esta narrativa. Es más, recientes estudios han señalado cómo esta narrativa de la Reconquista y el propio término de Reconquista surgen en los siglos XVIII y XIX para reinterpretar-podríamos decir incluso que reinventar- el pasado en clave nacional, con claros fines identitarios y legitimadores (Ríos, 2005). El estudio analiza las narrativas que los estudiantes generaban al ser preguntados por este período de la historia de su nación (López, Carretero y Rodríguez-Moneo, 2014a). Los participantes fueron estudiantes universitarios de la Facultad de Psicología de la Universidad Autónoma de Madrid. A lo largo de una entrevista individualizada, el participante generaba una narrativa sobre los hechos que habían ocurrido desde la llegada de los pueblos de origen musulmán a la Península Ibérica hasta la conquista de Granada por parte de los Reyes Católicos en 1492. Para llevar a cabo el análisis de las narrativas se analizaron los siguientes aspectos: el protagonista de la narrativa, el modo en el que utilizaban conceptos como España y españoles, el tipo de juicios morales emitidos y el uso del concepto de Reconquista. Es importante destacar que, para no influir en las respuestas de los participantes, en ningún momento el entrevistador mencionaba términos 
como España, españoles o Reconquista, salvo que el participante lo hubiera utilizado de manera espontánea previamente.

Los resultados de este estudio arrojan ideas interesantes en lo que se refiere al uso de las narrativas nacionales y a cómo los estudiantes entienden los conceptos de nación o identidad nacional. La gran mayoría de ellos reprodujo la narrativa de la Reconquista, justificando y argumentando que lo que se produjo fue una reconquista del territorio nacional español. Muchos de los estudiantes establecieron a España y a los españoles como los protagonistas de su narrativa, aplicando estos conceptos a un período histórico en el que la nación española ni siquiera existía. Incluso la mitad de ellos explícitamente se vinculaban con estos protagonistas utilizando términos como "nosotros" o "nuestro" para referirse a los españoles y su territorio. Expresiones como "En el 711 fuimos colonizados por los musulmanes" reflejan este vínculo identitario (López, et. al., 2014a, p.18). Un aspecto muy llamativo del estudio es la gran presencia de juicios morales que legitiman las acciones de los supuestos "españoles" y juzgan negativamente las de los musulmanes, que claramente constituyen el "otro" en la narración. En torno al $80 \%$ de los participantes entendió el proceso como una reconquista y utilizaba este término espontáneamente en sus argumentos. El siguiente fragmento de uno de los participantes ilustra el tipo de interpretaciones encontradas en el estudio:

Los árabes invaden un territorio que no es suyo. Durante más de siete siglos están intentando conquistar lo que es todo el territorio español y, los españoles, que en esencia es su territorio antes de que lleguen los árabes, reconquistan el territorio para volverlo a hacer suyo. (Juan, 25 años)

(López, et al. 2014a, p.18)

Este estudio muestra cómo muchos estudiantes construyen narrativas sobre el pasado apoyándose claramente en conceptos como la nación o la identidad nacional. El problema es que están entendiendo estos conceptos de un modo natural, como si hubiesen existido desde siempre y lo aplican a momentos históricos previos al surgimiento de la nación misma. Los vínculos identitarios que muestran y los juicios morales que emiten reflejan cómo han alcanzado objetivos de carácter identitario promovidos desde la historiografía tradicional romántica. Sin embargo, la mirada crítica hacia el pasado queda en un segundo plano.

Resultados similares han sido encontrados en estudios empíricos realizados en otros países como Argentina. Los estudiantes argentinos también muestran una fuerte influencia de elementos identitarios a la hora de interpretar contenidos históricos vinculados al pasado de su nación. Una extensa descripción del caso argentino y su comparación con el español puede verse en Carretero, López, González y Rodríguez-Moneo (2012). En un estudio realizado con participantes argentinos de 13 hasta 16 años de edad, Carretero y van Alphen (2014) encontraron una fuerte influencia de la narrativa maestra argentina en las interpretaciones sobre el proceso de independencia de su nación. De nuevo se encontraron identificaciones explícitas de los participantes con los protagonistas del pasado mediante el uso del "nosotros", en este caso para referirse a los argentinos. Este estudio reincide en la idea de que los estudiantes aplican características nacionales a eventos del pasado ocurridos incluso con anterioridad a la aparición de la nación. Los estudios llevados a cabo con estudiantes argentinos muestran una fuerte presencia de creencias que defienden la preexistencia de la identidad y el territorio argentino antes incluso de la formación de la nación argentina misma (Carretero y Kriger, 2008, 2011).

En otro estudio López, Carretero y Rodríguez-Moneo (2014b) analizan de nuevo las narrativas generadas por estudiantes universitarios españoles pero, a diferencia de todos los estudios previamente mencionados, utilizando un contenido histórico relativo a una nación distinta a la suya. De este modo, el estudio busca romper precisamente el vínculo identitario nacional entre el estudiante y el contenido histórico. Así, esta vez el contenido histórico utilizado no fue relativo a la historia de España, sino a la de Grecia. En este nuevo estudio, los estudiantes generaban una narrativa sobre la denominada "Ocupación" otomana de Grecia y su posterior independencia. 
Es interesante señalar que existen importantes semejanzas entre las interpretaciones históricas tradicionales de la denominada "Ocupación" otomana de Grecia y la "Reconquista" española. Estamos ante dos claros ejemplos de narrativas nacionales fundacionales: la griega y la española respectivamente. En ambas se describe la invasión del supuesto territorio nacional -a manos de los musulmanes en ambos casos- que se prolonga durante varios siglos, y que concluye con la recuperación del territorio por parte de sus "legítimos" poseedores. Los resultados del estudio sobre la "Ocupación" otomana muestran cómo los estudiantes universitarios españoles generan, ahora sí, una narrativa mucho más crítica que la que reflejaba el estudio sobre la "Reconquista". La mayoría de estudiantes en este nuevo estudio no emite juicios morales sesgados hacia ninguno de los grupos protagonistas. Es decir, juzgan de manera similar las conquistas, ya sean éstas realizadas por los otomanos o por los griegos. Del mismo modo, aparecen interpretaciones más críticas sobre el territorio en disputa. Para la mayoría, el territorio no pertenece de manera natural a ningún grupo, sino que va cambiando a lo largo del tiempo. Estas interpretaciones contrastan fuertemente con las encontradas en el estudio de la "Reconquista", donde la mayoría de estudiantes legitimaban una pertenencia atemporal del territorio de la Península Ibérica a los españoles. Parece, por tanto, que la ruptura del vínculo identitario entre el participante y el contenido histórico, permite una visión más distanciada y menos sesgada de la narrativa. Sin embargo, sí encontramos un aspecto que se mantiene inalterado en ambos estudios, y es el modo en el que los estudiantes entienden la identidad nacional. En ambos casos, la gran mayoría de estudiantes entienden la identidad nacional como un elemento atemporal que ha existido desde siempre. En el estudio sobre la "Ocupación" otomana, la identidad nacional griega se entiende como algo que de un modo u otro, siempre ha estado presente en esos territorios, tal y como ocurría con la identidad nacional española en la Península Ibérica.

Estos estudios ponen de manifiesto cómo muchos estudiantes interpretan el pasado de su nación de un modo similar al que lo hacen las narrativas nacionales. Es decir, la nación y la identidad nacional se entienden como elementos atemporales con los cuales se establece un vínculo emocional. Además, las acciones del propio grupo nacional son mayormente juzgadas de modo positivo, estableciéndose una narrativa de conflicto entre el "nosotros" nacional y el "otro" extranjero. Por lo tanto, parece que las narrativas nacionales no sólo están muy presentes en el modo en el que se transmite la historia, sino también en la manera en que las personas generan explicaciones sobre la misma.

\section{Reflexiones finales}

El formato narrativo constituye, como hemos visto, un elemento central en el ámbito de la historia. Pese a que no es el único formato a través del que podemos acercarnos al pasado, posee un enorme impacto en la producción de la historia, su transmisión -tanto en el ámbito formal como en el informal- y, finalmente, en su aprendizaje. Las narrativas nacionales parecen jugar un papel decisivo en cada uno de estos ámbitos. La propia historiografía, marcada en sus inicios por un fuerte contexto nacionalista, fue la principal promotora de la generación de estas narrativas nacionales más cercanas a leyendas y mitos que a interpretaciones críticas sobre el pasado. Sin embargo, desde finales del siglo XX, dentro de la propia historiografía se aboga por el desarrollo de una revisión crítica de estas narrativas y del fenómeno nacional, procurando en gran medida desmontar los mitos nacionales (Foster, 2012; VonBorries, 2009; Wineburg, 2001). Este nuevo enfoque trata de desarrollar un pensamiento histórico que entienda el fenómeno nacional como un fenómeno construido socialmente en un período reciente de la historia y que permita dar cabida a diferentes narrativas y puntos de vista en la mirada hacia el pasado. Ahora bien, la propia historiografía actual no está exenta de defensores de un uso identitario nacional de la historia. Por supuesto, los usos políticos de la misma tampoco (Nakou y Barca, 2010). La pretensión de una historia "científica", crítica y plural, por un lado, y la pretensión práctica, que busca fundamentalmente el desarrollo de una identidad nacional, constituyen los dos brazos de una balanza que representa una tensión 
irresoluble (López y Carretero, 2012). Las narrativas nacionales parecen decantar en muchas ocasiones el peso de dicha balanza en favor de los objetivos identitarios.

El presente artículo pretende arrojar algo de luz sobre el funcionamiento, la transmisión y el aprendizaje de estas narrativas nacionales. Si queremos seguir profundizando en un enfoque que permita el desarrollo de un pensamiento histórico, es relevante conocer cómo funcionan estas narrativas. Para ello, disciplinas como la psicología, la sociología o las ciencias políticas pueden aportar claves importantes. Este es el caso de la noción desarrollada por Wertsch (2004) relativa a los esquemas narrativos maestros. Las narrativas nacionales constituirían esquemas narrativos maestros que guían el recuerdo y la construcción de las narrativas específicas que generamos las personas a la hora de interpretar el pasado. Dicho de otro modo, las narrativas oficiales y sobre todo los conceptos de nación e identidad nacional, están en la base de muchas de las narrativas que se generan para dar sentido al pasado.

En cuanto a la transmisión del conocimiento histórico, son muchos los estudios que ponen de manifiesto cómo los contenidos nacionales y las narrativas nacionales todavía están muy presentes en los currículos de muchos países y en gran parte de sus libros de texto (Foster, 2012; Symcox y Wilschut, 2009; Van der Leeuw-Roord, 2009). Sin embargo, los libros de texto sólo constituyen un medio más a través de los cuales se transmiten estas narrativas nacionales. En este artículo se ha pretendido poner un fuerte énfasis en el papel que juegan otros mecanismos presentes en el ámbito informal a la hora de transmitir y reforzar las narrativas nacionales. Películas, monumentos, celebraciones nacionales, medios de comunicación, son sólo algunos de los canales que reivindican ese nacionalismo banal del que habla Billig (1995) y que en gran medida respaldan las narrativas nacionales.

Los recientes estudios realizados con estudiantes ponen de manifiesto el enorme peso que tienen estas narrativas nacionales en su comprensión del pasado. Estos estudios comienzan a darnos pistas sobre qué elementos caracterizan las interpretaciones de los estudiantes. Especialmente relevante parece ser el modo en el que entienden conceptos como el de nación e identidad nacional, conjuntamente con los juicios morales sesgados que emiten en sus narrativas. Desconocemos hasta qué punto los aprendizajes de los alumnos se ven más influenciados por el ámbito formal o por esas otras prácticas informales en las que se encuentran con el pasado. Si queremos tratar de desarrollar un pensamiento histórico en nuestros estudiantes que les permita distanciarse de objetivos identitarios nacionales y acercarse a la naturaleza construida del conocimiento histórico, se hace necesario seguir tratando de comprender el funcionamiento y la influencia de las narrativas nacionales. De este modo podremos inclinar un poco la balanza hacia perspectivas que desmitifiquen y desmonten estas narrativas, permitiendo desarrollar a nuestros alumnos habilidades para comprender el pasado desde un enfoque diferente. Un enfoque más plural, distanciado y que entiende la historia no como una narrativa verdadera, única e inmutable del pasado, sino como una mirada hacia el pasado plural -en muchos casos conflictiva- y en constante cambio.

\section{Bibliografía}

Alridge, D. P. (2006). The limits of master narratives in history textbooks: An analysis of representations of Martin Luther King, Jr. Teachers College Record, 108, 662-686.

Álvarez Junco, J. (2001). Mater dolorosa. La idea de España en el siglo XIX. Madrid: Santillana.

Anderson, B. (1983). Imagined communities: reflections on the origin and spread of nationalism. London: Verso.

Asensio, M. y Pol, E. (2012). From Identity Museums to Mentality Museums: Theoretical basis for history museums. En Carretero, M., Asensio, M. y Rodríguez-Moneo, M. (eds.), History Education and the Construction of National Identities (pp. 257-268). Charlotte CT: Information Age Publishing.

Aunión, J. A. (2012, 10 de Octubre). Wert quiere "españolizar" Cataluña. El País. Recuperado de http://sociedad.elpais.com 
Balibar, E. (1991). The nation form: history and Ideology. En Wallerstein, I. y Balibar, E. (eds.), Race, Nation, Class: ambiguous identities (pp. 86-106). London: Verso.

Ballantyne, T. (2005). Putting the nation in its place?: World history and C. A. Bayly's The birth of the modern world. In Curthoys, A. y Lake, M. (eds.), Connected Worlds: History in transnational perspective (pp. 23-44). Canberra: ANU E press.

Bartlett, F.C. (1932). Remembering: A study in experimental and social psychology. Cambridge: Cambridge University Press.

Barton, K. C. (2009). The denial of desire: How to make history education meaningless. En Symcox, L. y Wilschut, A. (eds.), International review of History education: National History Standars. The problem of the canon and the future of teaching History (pp. 265-282). Charlotte, NC.: Information Age Publishing.

Barton, K. C. y Levstik, L. (ed.). (2004). Teaching History for the Common Good. Mahwah, NJ: Lawrence Erlbaum Associates, Inc.

Barton, K. C. y McCully, A. W. (2005). History, Identity, and the School Curriculum in Northern Ireland: An Empirical Study of Secondary Students' Ideas and Perspectives. Journal of Curriculum Studies, 37(1), 85-116.

Berger, S., Eriksonas, L. y Mycock, A. (eds.) (2011). Narrating the nation: Representations in History, Media and the Arts. New York: Berghahn Books.

Billig, M. (1995). Banal Nationalism. London: Sage.

Bruner, J. (1990). Acts of Meaning. Cambridge, MA: Harvard University Press.

Carretero, M. (2011) Constructing patriotism. Teaching of history and memories in global worlds. Charlotte, CT: Information Age Publishing.

Carretero, M. y Bermúdez, A. (2012). Constructing Histories. En Valsiner, J. (ed.), Oxford Handbook of Culture and Psychology (pp. 625-646). Oxford: Oxford University Press.

Carretero, M. y Kriger, M. (2006). La usina de la patria y la mente de los alumnos. Un estudio sobre las representaciones de las efemérides escolares argentinas. En Carretero, M., Rosa, A. y González, M. F. (eds.), Enseñanza de la Historia y Memoria colectiva (pp. 169-196). Buenos Aires: Paidós.

Carretero, M. y Kriger, M. (2008). Narrativas históricas y construcción de la identidad nacional: representaciones de alumnos argentinos sobre el "Descubrimiento" de América. Cultura y Educación, 20(2), 229-242.

Carretero, M. y Kriger, M. (2011). Historical representations and conflicts about indigenous people as national identities. Culture and Psychology, 17(2), 177-195.

Carretero, M., Jacott, L. y López-Manjón, A. (2002). Mexican and Spanish history textbooks. Do students learn the same story? Learning and Instruction, 12, 651-665.

Carretero, M. y López, C. (2010). The Narrative mediation on historical remembering. En Salvatore, S., Valsiner, J., Simon, J. T. y Gennaro, A. (eds.), Yearbook of Idiographic Science (Vol. 3) (pp. 285-294). Roma: Firera y Liuzzo.

Carretero, M., López, C., González, M. F. y Rodríguez-Moneo, M. (2012). Students historical narratives and concepts about the nation. En Carretero, M., Asensio, M. y Rodríguez-Moneo, M. (eds.), History Education and the Construction of National Identities (pp. 153-170). Charlotte CT: Information Age Publishing.

Carretero, M. y Montanero, M. (2008). Enseñanza y aprendizaje de la Historia: aspectos cognitivos y culturales. Cultura y Educación, 20(2), 133-143.

Carretero, M. y Van Alphen, F. (2014). Do Master Narratives Change Among High School Students? A Characterization of How National History Is Represented. Cognition and Instuction, 32(3), 290-312.

Connor, W. (2004). The timelessness of nations. Nations and Nationalism, 10, 35-47.

Cruz Prados, A. (2005). El nacionalismo, una ideología [Nationalism, an ideology]. Madrid: Tecnos.

Duara, P. (ed.). (1995). Rescuing History from the Nation: Questioning Narratives of Modern China. Chicago: University of Chicago Press. 
Egan, K. (1997). The Educated Mind: How Cognitive Tools Shape Our Understanding. Chicago: The University of Chicago Press.

Finn, P. (2007, July 20). New manuals push a Putin's-eye view in Russian schools. The Washington Post. Recuperado de http://www.washingtonpost.com

Foster, S. (2006). Whose History? Portrayal of immigrant groups in U.S. History textbooks, 1800-Present. En Foster, S. J. y Crawford, K. A. (eds.), What shall we tell the children? International perspectives on school history textbooks (pp 155-178). Greenwich, CT: Information Age Publishing.

Foster, S. (2012). Re-thinking historical textbooks in a globalised world. En Carretero, M., Asensio, M. y Rodríguez-Moneo, M. (eds.), History Education and the Construction of National Identities (pp. 49-62). Charlotte CT: Information Age Publishing.

Gellner, E. (1983). Nations and nationalism. New York: Cornell University Press.

González de Oleaga, M. (2012). Historical narratives in the colonial, national and ethnic museums of Argentina, Paraguay and Spain. En Carretero, M., Asensio, M. y Rodríguez-Moneo, M. (eds.) History education and the construction of national identities (pp. 239-255). Charlotte, NC: Information Age Publishing.

Grever, M. (2006). Nationale identiteit en historisch besef. De risico's van een canon in de postmoderne samenleving [Identidad Nacional y Conciencia Histórica. Riesgos de un canon en una sociedad posmoderna]. Tijdschift voor Geschiedenis, 119, 160-177.

Grever, M. y Stuurman, S. (eds.) (2007). Beyond the Canon: History for the $21^{\text {st }}$ century. London: Palgrave Macmillan.

Hobsbawm, E. (1997). Nations and Nationalism since 1780: programme, myth, reality. Cambridge: Cambridge University Press.

Hobsbawm, E. y Ranger, T. (eds.). (1983). The Invention of Tradition. Cambridge: Cambridge University.

Hsiao, Y. (2005). Taiwanese Students' Understanding of Differences in History Textbook Accounts. En Ashby, R., Gordon, P. y Lee, P. (eds.), Understanding History. International review of History Education. Vol. IV (pp. 54-67). New York: Routledge.

Ichijo, A. y Uzelac, G. (eds.) (2005). When is the Nation? New York: Routledge.

Janmaat, J. G. (2005). Ethnic and Civic Conceptions of the Nation in Ukraine's History Textbooks. European Education, 37(3), 20-37.

López, C. y Carretero, M. (2012). Identity construction and the goals of history education. En Carretero, M., Asensio, M. y Rodríguez-Moneo, M. (eds.), History education and the construction of national identities (pp. 139-150). Charlotte, NC: Information Age Publishing.

López, C., Carretero, M. y Rodríguez-Moneo, M. (2014a). Conquest or Reconquest? Students' Conceptions of Nation Embedded in a Historical Narrative. Journal of the Learning Sciences, 24(2), 252-285.

López, C., Carretero, M. y Rodríguez-Moneo, M. (2014b). Telling a national narrative that is not your own. Does it enable critical historical consumption? Culture y Psychology, 20(4), 547-571.

Lorenz, C. (2011). Drawing the line: 'Scientific' history between Myth-making and Myth-breaking. En Berger, S., Eriksonas, L. y Mycock, A. (eds.), Narrating the nation: Representations in History, Media and the Arts (pp. 35-55). New York: Berghahn Books.

Mak, G. (2005). Gedoemd tot kwetsbaarheid [Doomed to vulnerability]. Amsterdam: Atlas.

Mandler, J. M. (1984). Stories, scripts and scenes: Aspects of schema theory. Hillsdale, NJ: Lawrence Erlbaum Associates.

Nakou, I. y Barca, I. (eds.) (2010). Contemporary public debates over history education. International Review of History Education Series. Charlotte, NC: Information Age Publishing.

Paxton, R. J. (1999). A Deafening Silence: History Textbooks and the Students Who Read Them. Review of Educational Research, 69(3), 315-339.

Pérez Garzón, J. S. (2001). Nación española y revolución liberal: la perspectiva historiográfica de los coetáneos [Spanish nation and liberal revolution: historiographical perspective of contemporary 
people]. En Forcadell, C.y Peiró, I. (eds.). Lecturas de la Historia: Nueve reflexiones sobre la Historia de la Historiografía (pp. 23-54). Zaragoza: Institución Fernando el Católico.

Renan, E. (1882). What is a Nation? En Bhabha, H. K. (1990) (ed.), Nation and Narration (pp. 8-22). Routledge: London.

Ríos Saloma, M. F. (2005). From the Restoration to the Reconquest: The construction of a national myth (An historiographical review. $16^{\text {th }}-19^{\text {th }}$ centuries). España medieval, 28, 379-414.

Ross, M. (1989). Relation of Implicit Theories to the Construction of Personal Histories. Psychological review, 96(2), 341-357.

Rüsen, J. (2004). Historical Consciousness: narrative structure, moral function, and ontogenetic development. En Seixas, P. (ed.), Theorizing historical consciousness (pp. 63-85). Toronto: University of Toronto Press.

Smith, A. D. (1991) National Identity. London: Penguin.

Symcox, L. y Wilschut, A. (2009). Introduction. En Symcox, L. y Wilschut, A. (eds.), National History Standars. The problem of the canon and the future of teaching History. International review of History education (Vol. 5. pp. 1-11). Charlotte, NC.: Information Age Publishing.

Valsiner, J. (2012). Monuments in our minds: Historical symbols as cultural tools. En Carretero, M., Asensio, M. y Rodríguez-Moneo, M. (eds.), History education and the construction of national identities (pp. 327-346). Charlotte, CT: Information Age Publishing.

Van der Leeuw-Roord, J. (ed.) (2004). History changes. Facts and figures about history education in Europe since 1989. The Hague: EUROCLIO.

Van der Leeuw-Roord, J. (2007). Two Steps Forward, One Step Back: Shoring Our Stories and Looking for the Common Threads. En Smart, D. (ed.), Making a Difference: Fifteen years of EUROCLIO (pp. 66-73). The Hague: EUROCLIO.

Van der Leeuw-Roord, J. (2009). Yearning for yesterday. Efforts of history professionals in Europe at designing meaningful and effective school History curricula. En Symcox, L. y Wilschut, A. (eds.), National History Standards. The problem of the canon and the future of teaching History. International review of History education (Vol. 5. pp. 73-94). Charlotte, NC.: Information Age Publishing.

VanSledright, B. (2008). Narratives of Nation-State, Historical Knowledge, and School History Education. Review of Research in Education, 32(1), 109-146.

Vasagar, J. (2012, November 24). Michael Gove accuses exam system of neglecting British history. The Guardian. Recuperado de http://www.guardian.co.uk

Von Borries, B. (2009). Competence of historical thinking, mastering of a historical framework, or knowledge of the historical canon? En Symcox, L. y Wilschut, A. (eds.), National history standards: the problem of the canon and the future of teaching history (pp.283-306). USA: Information Age Publishing.

Wallerstein, I. (1991). The construction of peoplehood: racism, nationalism, ethnicity. En Wallerstein, I. y Balibar, E. (eds.), Race, Nation, Class: ambiguous identities (pp. 71-85). London: Verso.

Wertsch, J. V. (2004). Specific narratives and Schematic narrative templates. En Seixas, P. (ed.), Theorizing historical consciousness (pp. 49-62). Toronto: University of Toronto Press.

Wineburg, S. (2001). Historical Thinking and Other Unnatural Acts. Philadelphia: Temple University Press.

Wineburg, S., Mosborg, S. y Porat, D. (2001). What Can Forrest Gump Tell Us about Students' Historical Understanding? (motion picture). Social Education, 65(1), 55.

Wineburg, S., Mosborg, S., Porat, D., y Duncan, A. (2007). Common Belief and the Cultural Curriculum: An Intergenerational Study of Historical Consciousness. American Educational Research Journal, 44, 40-76. 



\section{Panta Rei}

PANTA REI es una revista digital de investigación orientada a la Historia y otras ciencias afines. Su principal objetivo es la transmisión del conocimiento científico, dando una oportunidad también a los jóvenes investigadores que quieren abrirse camino en el estudio de las ciencias humanas y sociales. Se compone de estudios originales relacionados con la disciplina histórica así como su didáctica y difusión. Las diferentes secciones que componen la revista son: artículos de investigación, entrevistas a profesionales, recensiones de monografías de actualidad y crónicas de congresos o eventos científicos relevantes.

Todos los artículos publicados son objeto de un proceso de revisión a cargo de un mínimo de dos evaluadores, que se consideran expertos en el ámbito temático del artículo propuesto. Nuestro deseo es poder ofrecer unos contenidos rigurosos, de calidad y de interés.

EI CEPOAT (Centro de Estudios del Próximo Oriente y la Antigüedad Tardía de la Universidad de Murcia) es la institución encargada de la coordinación y gestión de la revista, desde donde anualmente se lanzará la convocatoria para aquellos que estén interesados en publicar sus trabajos, siempre relacionados con la Historia, Arqueología, Historia del Arte, Didáctica de las Ciencias Sociales, etc.

PANTA REI is a digital journal focused on History and other sciences related to it. Its main objective is the transmission of scientific knowledge by giving also an opportunity to young researchers who want to make their way in the study of human and social sciences. It is composed by original studies related to History, as well as its didactics and promotion. The different sections of this journal are: research articles, interviews to professionals, recensions on monographs about current issues and reports about congresses or relevant scientific events.

All the articles published are subject to a revision process carried out by a minimum of two reviewers who are considered to be experts in the field of the article proposed. Our wish is to offer rigorous contents with quality and being of interest to the reader.

CEPOAT (Centre of Studies of the Middle East and Late Antiquity of the University of Murcia) is the institution in charge of the coordination and management of this journal. This is the centre from where the call for papers will be launched for all the people interested in publishing their papers, always related to History, Archeology, Art History, Didactics of the Social Sciences, etc. 


\section{Normas de Publicación}

El autor se compromete a enviar trabajos originales, que no se encuentren publicados en otras revistas ni en otros idiomas. Así mismo, el mismo artículo no podrá ser presentado en otras revistas mientras dure el proceso de evaluación.

\section{Envío y presentación de originales}

Los artículos se enviarán exclusivamente a través del correo electrónico a la dirección pantarei@um.es. Los textos serán enviados en formato DOC y las imágenes en formato JPEG o TIFF, y con un tamaño mínimo de 2000 px. Éstas no aparecerán incorporadas en el texto, sino enviadas en archivo aparte y correctamente numeradas según su posición en el texto. Junto al trabajo, se rellenará y enviará un documento aparte en el que se especifiquen los datos del autor siguiendo el modelo disponible en la página Web de la revista.

Para la redacción de los trabajos se tendrá en cuenta el Manual de la American Psychological Association, en su sexta edición. La extensión máxima de los trabajos será de 30 páginas. La tipografía será Arial 11, con interlineado sencillo y sin espacio alguno entre párrafos. El texto deberá ir justificado a ambos márgenes y sin sangría en los primeros párrafos. Los márgenes serán de $2,50 \mathrm{~cm}$. En los casos en los que fuera necesario incorporar notas, éstas irán a pie de página, enumeradas consecutivamente, con tipografía Arial 10, interlineado sencillo y justificadas a ambos márgenes.

Una información más detallada se encuentra disponible en la página http://www.um.es/cepoat/ pantarei.

\section{Proceso de valoración y evaluación}

Una vez recibidos los trabajos, la Revista realizará una primera valoración. Si el trabajo enviado se ajusta a las normas de presentación propuestas, la temática es coincidente con la línea editorial de la revista y posee la calidad científica necesaria, será remitido al consejo asesor para una primera evaluación. Si no es así en este primer paso se puede rechazar directamente los documentos que incumplan claramente la línea editorial.

Será el Consejo Asesor quien indique a la revista la originalidad, relevancia, estructura, redacción, aparato bibliográfico, etc. del trabajo enviado y, para ello, se designará a dos revisores expertos externos que evaluarán cada uno de los trabajos, que pueden formar parte (o no) de este Consejo Asesor. La selección de los revisores se ajustará a la temática y características metodológicas del trabajo. El nombre y filiación de los autores serán eliminados del trabajo para su revisión, así como los revisores actuarán de manera anónima y confidencial.

Los revisores deberán rellenar un informe de evaluación que centrará su atención en aspectos tales como características formales, originalidad y novedad de los trabajos, relevancia de las propuestas y los resultados, calidad metodológica y validez científica.

Una vez terminado el proceso se decidirá la aceptación o no de los mismos y su publicación en el número que sea pertinente, así como las modificaciones susceptibles de ser realizadas para su final publicación. Dicha notificación se enviará únicamente por correo electrónico, en un plazo máximo de seis meses. 


\section{Publishing rules}

The author is committed to submit original papers not having been published in other reviews or in other languages. In this way, it is not allowed for the same paper to be presented in other reviews during the evaluation process.

\section{Submission and presentation of originals}

The articles will be exclusively submitted by email to pantarei@um.es. The texts will be submitted in DOC format and the images in JPEG or TIFF format, and with a minimum size of 2000 px. Images will not be integrated in the text but sent in another file and properly numbered according to their position in the text. Attached to the paper, a document will be filled out and sent where the author's data will be specified following the model available on the website.

The sixth edition of the Manual of the American Psychological Association will be taken into account for the writing of the papers. The length of the papers must not exceed 30 pages. Typography will be Arial 11 , with simple line spacing and no space between paragraphs. The text must be justified on both margins without indentation in the first paragraphs. Margins size will be $2.50 \mathrm{~cm}$. Where it could be necessary the incorporation of notes, they will be at the bottom of the page, consecutively numbered with typography Arial 10, simple line spacing and justified on both margins.

More detailed information is available on the website: http://www.um.es/cepoat/pantarei.

\section{Examination and assessment process}

The Journal will submit the papers to a first examination once received. If the paper follows the presentation guidelines, the subject agrees with the editorial line of this journal, and possess the scientific quality required, it will be sent to the advisory council for a first assessment. If not, the documents which clearly fail to complete the editorial line may be rejected straightaway in this first step.

The Advisory Council will indicate the originality, relevance, structure, writing, bibliography, etc. of the text to the journal; for this purpose, two outside experts will be designated to review the papers; these experts can be (or not) part of this Advisory Council. The selection of the experts will adjust to the subject and methodological characteristics of the paper. Name and affiliation of the author will be eliminated from the text for its review, in this way experts will act anonymously and confidentially.

The experts will fill out an assessment report which will focus on aspects such as formal characteristics, originality and novelty of the papers, relevance and results of the proposal, methodological quality and scientific validity.

Once the process is finished, the acceptance or not of the papers and its publication in the corresponding edition will be decided, as well as the modifications that may be done for its final publication. This notification will be sent by email within 6 months maximum. 

